\title{
Ueber das Schicksal einiger isomeren Oxychinoline (Carbostyril und Kynurin) im Thierkörper. \\ Von
}

Dr. Béla v. Fenyressy,

Assistenten am pharmacolog. Institut der Universität zu Budapest.

(Aus der chemischen Abtheilung des physiologischen Institutes in Berlin.)

(Der Redaction zugegangen am 12. August 1900.)

Die ersten Untersuchungen über das Verhalten einiger Chinolinderivate im Thierkörper wurden im Zusammenhange mit der Frage über die Entstehung der Kynurensäure, einer im normalen Hundeharn vorkommenden Oxychinolincarbonsäure, vorgenommen. Die Versuche, die unter Jaffe's Leitung von A. Schmidt) und F. Rosenhain ${ }^{2}$ ) ausgeführt und in deren Verlaufe auch die in dieser Arbeit zu behandelnden isomeren Oxychinoline berücksichtigt wurden, führten in Bezug auf die gestellte Frage zu keinem positiven Resultate, da Bildung von Kynurensäure nach Verabreichung einer Reihe verwandter Substanzen nicht beobachtet werden konnte. R. Cohn ${ }^{8}$ ) nahm die physiologisch-chemische Untersuchung dreier isomerer Methylchinoline (Chinaldin, Ortho- und Paramethylchinolin) in der Erwartung vor, dass dieselben Synthesen mit Produkten des thierischen Stoffwechsels eingehen würden. Dies war aber nicht der Fall, und der Verfasser gelangt zu

1) A. Schmidt. Ueber das Verhalten einiger Chinolinderivate im Thierkörper mit Rücksicht auf die Bildung von Kynurensäure. Inaug.Dissert. Königsberg 1884.

2) F. Rosenhain. Beitr. z. Kenntniss der Kynurensäurebildung. im Thierkörper. Inaug.-Dissert. Königsberg 1886.

3) R. Gohn. Zeitschr. f. physiol. Chem. Bd. XX, S. 210. 
der Annahme, dass der Chinolinkern im Thierkörper besonders leicht zerstört werden kann. Dass diese These keine allgemeine Gültigkeit für sonstige Chinolinderivate hat, wie es übrigens schon Cohn vermuthete, dafür wurde der Beweis durch die Untersuchungen von E. Rost und von G. Brahm über das physiologisch-chemische Verhalten des Chinosols (ein Gemenge von o-Oxychinolinsulfat und Kaliumsulfat) erbracht. Rost $^{1}$ ) wies nach, dass das 0-Oxychinolin im Thierkörper eine Paarung mit Schwefelsäure eingeht. $\mathrm{Brahm}^{2}$ ) gelang es, aus dem Harne der mit Chinosol gefütterten Hunde und Kaninchen o-Oxychinolinglykuronsäure $\mathrm{zu}$ gewinnen.

Diese letztere Arbeit gab die Veranlassung zu weiteren Untersuchungen über das Schicksal isomerer Oxychinoline im Thierkörper.

Das Chinolin ist bekanntlich aus einem Benzol- und einem Pyridinninge zusammengesetzt.

Da das von Rost und Brahm untersuchte Chinolinderivat im Benzolkern hydroxylirt war, so lag es am nächsten, isomere Körper, die aber die OH-Gruppe im Pyridinkern tragen, auf ihr physiologisch-chemisches Verhalten zu prüfen. Dieser Aufgabe kam ein weiteres Interesse in Folge der nahen Verwandtschaft eines der Py-Oxychinoline zur Kynurensäure zu.

Aus diesem Grunde wurden nachstehende Untersuchungen über das Verhalten von Carbostyril und Kynurin im Thierkörper auf Vorschlag und unter Leitung von Herrn Prof. Thierfelder aufgenommen.

\section{Carbostygril.}

$\mathrm{C}_{9} \mathrm{H}_{7} \mathrm{NO}\left(+\mathrm{H}_{2} \mathrm{O}\right)$. - Das nöthige Material wurde von der chemischen Fabrik Th. Schuchardt (Görlitz) bezogen. Das Präparat erwies sich als völlig rein. (Schmelzpunkt: $198-200^{\circ}$ C.)

Ueber das Schicksal des Carbostyrils im Thierkörper gibt A. Schmidt ${ }^{3}$ ) auf Grund zweier Kaninchen-Versuche Folgen-

1) E. Rost, Arbeiten aus dem Kaiserl. Gesundheitsamte. 15, 288.

2) C. Brahm, Ueber das Chinosol, sein Verhalten im Thierkörper etc. Zeitschr. f. physiol. Chemie, Bd. XXVIII, S. 439.

3) l. c. 
des an: «Der Urin gab in beiden Fällen keine Spur von Kynurensäure, dagegen schien er eine gepaarte Schwefelsäure zu enthalten.» Es wurden je 0,25 g Carbostyril einmal per os, das andere Mal subcutan verabreicht. Giftwirkung wurde nicht beobachtet.

Nachstehende Versuchsergebnisse wurden ebenfalls an Kaninchen gewonnen. Die Thiere wurden mit Hafer ernährt, das Carbostyril wurde in der Regel (die wenigen subcutanen Versuche werden besonders besprochen) per os, und zwar wegen der Schwerlöslichkeit der Substanz in Form von Gummiemulsion zugeführt. Die einmalige Dosis war gewöhnlich 0,5 g (resp. $0,25-0,30$ pro $1000 \mathrm{~g}$ Körpergewicht). Hierbei traten regelmässig Vergiftungserscheinungen auf; doch waren dieselben gewöhnlich bald überwunden, so dass es zweckmässig erschien, die ohnehin geringe Ausbeute an dem zu besprechenden Harnprodukt nicht durch Einschränkung der Carbostyrilzufuhr noch weiter herabzudrücken.

Die Wirkung des Carbostyrils äusserte sich in Form von Lähmungserscheinungen. Schon nach 5-10 Minuten nach der Eingabe zeigten die Thiere auffallende Mattigkeit, und nach etwa einer halben Stunde fand man sie mit ganz schlaffen Extremitäten- und Nackenmuskulatur gewöhnlich in Seitenlage; in Rückenlage versetzt, konnten sie sich nicht aufrichten; auf stärkere Reize zeigten die Thiere machtlose Abwehrversuche; Reflexe waren erhalten. Die Athmung, Anfangs beschleunigt, war in diesem Stadium der Lähmung sehr verlangsamt und erschwert. Seitens der Circulation wurden, ausser einer anfänglichen, nicht sehr starken Erweiterung der Ohrgefässe, keine auffälligen Erscheinungen beobachtet. Aus dem beschriebenen Lähmungszustande erholten sich die Thiere gewöhnlich in 3-5 Stunden und verhielten sich dann bis zum nächsten Tage scheinbar ganz normal. Erst bei längerer Behandlung stellte sich eine Abnahme der Fresslust und des Körpergewichtes, sowie ein schwererer Verlauf der Einzelvergiftungen ein. Sodann wurde die Verabreichung von Carbostyril auf einige Tage ausgesetzt, was ohne Zeitverlust geschehen konnte, da gleichzeitig an 3-5 Thieren experimen- 
tirt wurde. Tödtlicher Verlauf wurde entweder nach einmaligen zu grossen Dosen oder aber an schon vorbehandelten Thieren auch nach der gewöhnlichen Gabe beobachtet. In solchen Fällen verblieben die Thiere bis zum Tode 12-24 Stunden lang in dem schweren Lähmungszustande. Der Sectionsbefund war wenig charakteristisch. Als constanter Befund sollen ausgedehnte Blutungen im Magenfundus hervorgehoben werden.

Einen näheren Einblick in das Wesen der Carbostyrilwirkung gestatteten einige Froschversuche. Frösche, denen man 0,05-0,10 g Carbostyril in Pulverform in den Magen bringt, zeigen bald dieselben Lähmungserscheinungen, die an den Kaninchen beobachtet wurden. Das Herz eines vollständig gelähmten Frosches schlägt noch viele Stunden lang mit normaler Kraft und Frequenz. An zwei solchen Thieren wurde die Prüfung der elektrischen Erregbarkeit des Plexus ischiadicus und der Schenkelmuskulatur mit Hülfe des Schlittenapparates vorgenommen. Es zeigte sich nun, im Vergleich mit einem normalen Thiere, eine sehr beträchtliche Abnahme der Wirksamkeit der Nervenreizung, dagegen eine ganz geringe oder auch keine Abnahme der direkten Muskelerregbarkeit.

Somit scheint das Carbostyril eine curareähnliche Wirkung zu besitzen.

Bezüglich der beschriebenen Magenblutungen soll erwähnt werden, dass Carbostyril, in Pulverform in das Auge eines. Kaninchens gebracht, eine ziemlich starke, wenn auch nur wenige Stunden lang dauernde Röthung der Bindehaut hervorruft.

Der Harn der mit Hafer ernährten Kaninchen ist concentrirt und stark sauer. Am ersten bis zweiten Tage nach Verabreichung von Carbostyril erschien im Harn oft rechtsdrehender Zucker. Derselbe wurde durch das optische Verhalten, durch die Trommer'sche Probe sowie durch die Gährungsfähigkeit charakterisirt. Nach der Vergährung, sowie an den weiteren Carbostyriltagen auch ohne Vorbehandlung zeigte der. Harn eine deutliche Linksdrehung, hielt ziemlich viel alka- 
lisches Kupferoxyd in Lösung, reducirte aber auch beim Kochen nicht.

Zur Reindarstellung der linksdrehenden Substanz diente das bekannte Bleiverfahren. Der Harn wurde zunächst mit neutralem, sodann mit basischem Bleiacetat gefällt. Dieser letztere Niederschlag wurde nach Auswaschen mit heissem Wasser im $\mathrm{H}_{2} \mathrm{~S}-\mathrm{S}$ trom entbleit, das Filtrat auf dem Wasserbade eingeengt; bei geeigneter Concentration schieden sich aus der wiederholt mit Thierkohle behandelten Flüssigkeit voluminöse, aber specifisch leichte Krystallmassen ab, welche durch Umkrystallisiren aus heissem Wasser leicht gereinigt- werden konnten. Es wurden nach Verabreichung von ungefähr $80 \mathrm{~g}$ Carbostyril etwa $4 \mathrm{~g}$ dieser Substanz gewonnen.

Die Substanz, eine schneeweisse, weiche Masse besteht aus mikroskopisch kleinen, sternförmig angeordneten Krystallnadeln. Die Substanz hat keinen scharfen Schmelzpunkt; von $220^{\circ} \mathrm{C}$. an färbt sie sich allmählich dunkler und bei 250 bis $252^{\circ}$ C. verkohlt sie. Sie ist schwer löslich in kaltem, leicht in heissem Wasser, fast unlöslich in Alkohol, Aether, Chloroform, Benzol etc. In Alkalien löst sich die Substanz sehr leicht auf und wird aus einer concentrirten alkalischen Lösung auf Zusatz von wenig $\mathrm{HCl}$ krystallinisch gefällt; in überschüssiger HCl löst sie sich jedoch wieder auf. Die wässerige Lösung der Substanz reagirt sauer, hält viel alkalisches Kupferoxyd in Lösung, reducirt aber beim Kochen nicht. Auf Grund der beobachteten linksdrehenden Eigenschaft des Harns wurde eine gepaarte Glycuronsäure vermuthet und die Spaltung derselben versucht. Gegen Einwirkung von heissen Mineralsäuren erwies sich die Substanz als sehr widerstandsfähig. Erst nach 15-20 Minuten lang fortgesetztem Kochen mit 100,0iger $\mathrm{HCl}$ gelingt die Reductionsprobe in Spuren, wogegen die von $\mathrm{Brahm}$ isolirte 0 -Oxychinolinglycuronsäure bei derselben Behandlung schon nach 5 Minuten ausgiebige Spaltung erfährt. Zur Prüfung des optischen Verhaltens eignet sich die Substanz wegen ihrer Schwerlöslichkeit nicht. Die angegebenen Eigenschaften der Substanz machten es in hohem Grade wahrscheinlich, dass wir es mit einer gepaarten Gly- 
curonsäure zu thun haben. Zur vollen Sicherheit war die Analyse der Substanz nöthig.

Die qualitative Untersuchung der Säure ergab die Anwesenseit von $\mathrm{C}, \mathrm{H}$ und $\mathrm{N}$, aber keinen $\mathrm{S}$.

Zum Zwecke der Elementaranalyse wurde die Substanz im Vacuum über $\mathrm{H}_{2} \mathrm{SO}_{4}$ bis zur Gewichtsconstanz getrocknet, wobei nur eine ganz geringe Gewichtsabnahme stattfand; eine weitere Gewichtsabnahme trat bei $100^{\circ}$, endlich auch bei $140^{\circ}$ nicht ein.

Bei der Verbrennung der Substanz gelang es uns nicht, nur irgendwie übereinstimmende Resultate zu erzielen. Wir verzichteten auf weitere Versuche, da wir uns auf Grund der Erfahrungen von Brahm von der Analyse des Kalisalzes mehr Erfolg versprechen konnten.

Der N-Gehalt der freien Säure wurde nach Kjeldahl mit der Kr üger'schen Modification bestimmt. Die Substanz wurde bei $100^{\circ}$ bis zur Gewichtsconstanz getrocknet.

I. $0,1603 \mathrm{~g}$ Säure verbrauchten $4,50 \mathrm{ccm}$. 1/10 N.-HCl,

II. 0,1963 > > $5,50>1 / 10$,
Gefunden $\mathrm{N}$
I. $3,93 \%$ $\mathrm{C}_{15} \mathrm{H}_{17} \mathrm{NO}_{8}$ verlangt
II. $3,92 \%$

Einen weiteren Aufschluss über die Zusammensetzung der Substanz erhielten wir durch Titration einer heissen Säurelösung mit ${ }^{1 / 10} \mathrm{~N}$.-KOH.

I. $0,1317 \mathrm{~g}$ Säure brauchten zur Neutralisation $3,9 \mathrm{ccm}$. 1/10 N.-KOH

II. 0,5592 > > > > $16,25>1 / 10$ >
$100 \mathrm{~g}$ Säure verbrauchen $\mathrm{g} \mathrm{K}$ Gefunden
I. 11,54 $\mathrm{C}_{15} \mathrm{H}_{17} \mathrm{NO}_{8}$ verlangt
II. 11,33 11,50

\section{Kalisalz.}

Dasselbe wurde theils durch Umsetzung des Barytsalzes (bereitet durch Neutralisiren der heissen Säurelösung mittelst $\mathrm{Ba}(\mathrm{OH})_{2}$ und Entfernen des überschüssigen $\mathrm{Ba}$ durch $\mathrm{CO}_{2}$ ) mit Hülfe von Kaliumsulfat, theils durch Neutralisiren der freien Säure mit KOH hergestellt. In beiden Fällen wurde die 
Lösung des Kalisalzes zunächst auf dem Wasserbade, dann im Vacuum über $\mathrm{H}_{2} \mathrm{SO}_{4}$ eingeengt. Krystallisation konnte aber auf diese Weise nicht, sondern erst durch Zusatz von Alkohol zur heissen Salzlösung bei nachträglichem Einengen im Vacuum erzielt werden.

Das Kalisalz krystallisirt in schwefelgelben Nadeln, ist leicht löslich in kaltem, noch mehr in heissem Wasser, unlöslich in Alkohol, Aether, Chloroform etc. Die Lösung reagirt neutral und dreht die Polarisationsebene stark nach links. Aus einer concentrirten Lösung wird die Säure durch Mineralsäuren freigemacht und krystallinisch abgeschieden.

Das Kalisalz hat, wie die freie Säure, keinen scharfen Schmelzpunkt. Es verkohlt bei $270-272^{\circ} \mathrm{C}$. Die im Vacuum über Schwefelsäure getrocknete Substanz bleibt bei $100-120^{\circ} \mathrm{C}$. unverändert.

Zum Zwecke der Analyse wurde das Kalisalz bei $100^{\circ}$ bis zur Gewichtsconstanz getrocknet. Die Substanz erwies sich sowohl bei der Verbrennung, wie auch beim Abrauchen mit $\mathrm{H}_{2} \mathrm{SO}_{4}$ als sehr schwer zerstörbar.

Die C-, H-Bestimmung wurde in geschlossenem Rohre mit Bleichromat unter nachträglicher 0-Durchleitung, N-Bestimmung nach Krüger ausgeführt. Um den K-Gehalt zu ermitteln, mussten wir, da das Zerstören des Salzes immer unter Zurücklassung von Kohle erfolgte und allzu geringe Werthe lieferte, einen Umweg einschlagen; wir lösten die abgewogene Menge des Kalisalzes in möglichst wenig Wasser, tügten einige Tropfen verdünnter $\mathrm{HCl}$ hinzu, entfernten die abgeschiedene organische Säure durch Filtriren und bestimmten aus dem in Lösung befindlichen $\mathrm{KCl}$ das $\mathrm{K}$ nach Abdampfen des Wassers in der üblichen Weise als $\mathrm{K}_{2} \mathrm{SO}_{4}$.

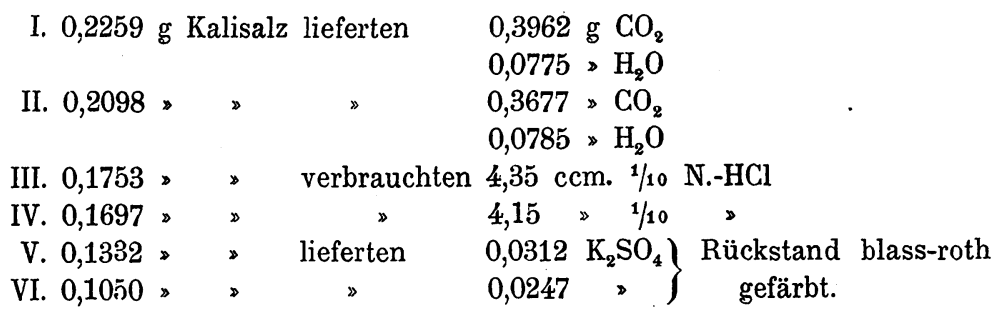




\begin{tabular}{cccccccc} 
& \multicolumn{9}{c}{ Gefunden $\%$} & & & $\mathrm{C}_{15} \mathrm{H}_{16} \mathrm{NO}_{8} \mathrm{~K}$ \\
verlangt 1$)$ \\
C & 47 & II & III & IV & V & VI & \\
$\mathrm{H}$ & 3,82 & 47,78 & - & - & - & - & $47,75 \%$ \\
$\mathrm{~N}$ & - & -16 & - & - & - & - & $4,24 \%$ \\
$\mathrm{~K}$ & - & - & 3,48 & 3,43 & - & - & $3,71 \%$ \\
& - & - & - & 10,49 & 10,54 & $10,37 \%$
\end{tabular}

Es handelt sich also hier um eine Carbostyril-Glycuronsäure (resp. um ihr Kalisalz), der auf Grund der Analysen die Formel $\mathrm{C}_{15} \mathrm{H}_{17} \mathrm{NO}_{8}$ (resp. $\mathrm{C}_{15} \mathrm{H}_{16} \mathrm{NO}_{8} \mathrm{~K}$ ) zukommt. Die Vereinigung ist also scheinbar ohne Austritt von $\mathrm{H}_{2} \mathrm{O}$ verlaufen. Man wird annehmen müssen, dass die Aldehydgruppe der Glycuronsäure zunächst in eine zweiwerthige Alkoholgruppe übergeführt, diese mit dem Carbostyril unter Austritt von 1 Mol. $\mathrm{H}_{2} \mathrm{O}$ zusammengetreten und keine innere Anhydridbildung erfolgt ist. Aehnlich erklärt Blum²) die Zusammensetzung der Dichlorthymolglycuronsäure.

Specifische Drehung des Kalisalzes.

\begin{tabular}{c|c|c|c|c|c|c}
\hline \hline & $\begin{array}{c}\text { Gehalt an } \\
\text { Substanz in } \\
100 \mathrm{ccm} .\end{array}$ & $\begin{array}{c}\text { Gehalt an } \\
\text { Substanz in } \\
100 \mathrm{~g}\end{array}$ & $\begin{array}{c}\text { Specifisches } \\
\text { Gewicht der } \\
\text { Lösung }\end{array}$ & $\begin{array}{c}\text { Länge } \\
\text { des Rohrs }\end{array}$ & $\begin{array}{c}\text { Beobachtete } \\
\text { Winkel. } \\
\text { drehung }\end{array}$ & $\begin{array}{c}\text { Specifische } \\
\text { Drehung } \\
{[\alpha] \mathrm{D}}\end{array}$ \\
\hline 1 & 4,8192 & 4,8190 & $\begin{array}{c}1,019 \\
\left(18^{\circ} \mathrm{C} .\right)\end{array}$ & 2,2 & $-9,20$ & $-85,17$ \\
\hline 2 & 2,3644 & 2,3680 & $\begin{array}{c}1,010 \\
\left(22^{\circ} \mathrm{C} .\right)\end{array}$ & 2,2 & $-4,30$ & $-81,55$ \\
\hline 3 & 1,1822 & 1,1840 & $\begin{array}{c}1,006 \\
\left(22^{\circ} \mathrm{C} .\right)\end{array}$ & 2,2 & $-1,93$ & $-73,52$ \\
\hline
\end{tabular}

Wie aus obiger Tabelle ersichtlich, nimmt das specifische Drehungsvermögen des Kalisalzes mit verminderter Concentration der Lösung ab.

1) Auf Veranlassung von Herrn Professor Thierfelder füge ich folgende Berichtigung ein: In der Arbeit von C. Brahm, Zeitschr. f. physiol. Chemie, Bd. XXVIII, S. 443, muss es nicht heissen:

$\begin{array}{cc}\mathrm{C}_{15} \mathrm{H}_{14} \mathrm{NO}_{7} \mathrm{~K} \text {, sondern } \mathrm{C}_{15} \mathrm{H}_{14} \mathrm{NO}_{7} \mathrm{~K} & 4 \mathrm{H}_{2} \mathrm{O} \\ 47,74 & 47,74 \\ 4,45 & 4,24 \\ 3,71 & 3,71 \\ 10,03 & 10,37\end{array}$

2) F. Blum, Ueber Thymolglycuronsäure. Zeitschr. f. physiol. Chemie, Bd. XVI, S. 514. 
Nachdem es sich herausstellte, dass sich das Carbostyril bezüglich der Paarung mit Glycuronsäure dem 0-0xychinolin ähnlich verhält, war es schon vorneherein zu vermuthen, dass es auch mit Schwefelsäure eine Synthese eingeht.

Dies wurde durch quantitative Bestimmung der vor und nach der Carbostyrilzufuhr ausgeschiedenen Harnschwefelsäuren bewiesen. Folgender Versuch wurde an einem hungernden Kaninchen ausgeführt. Erster Normaltag $=$ zweiter Hungertag.

Doppelte Bestimmungen der $\mathrm{H}_{2} \mathrm{SO}_{4}$ nach Baumann.

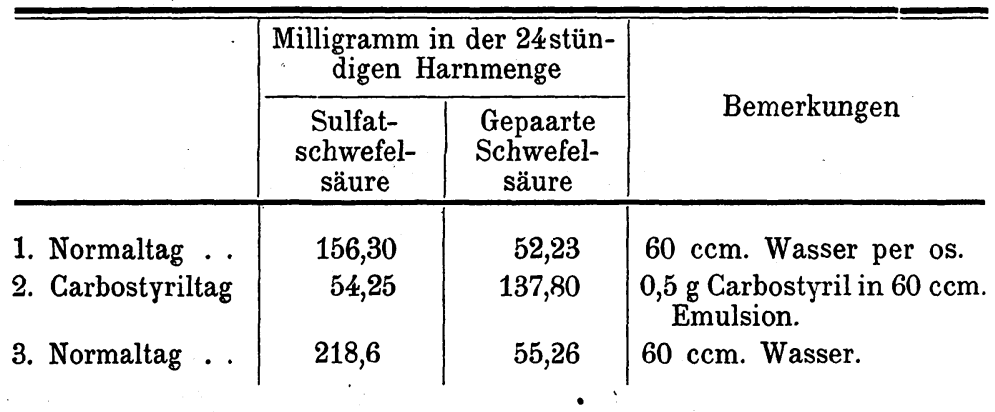

Die bedeutende Zunahme der gepaarten und die entsprechende Abnahme der Sulfatschwefelsäuren nach Verabreichung von Carbostyril weist auf die Bildung von Carbostyrilschwefelsäure hin. Aus den obigen Zahlen lässt es sich berechnen, dass etwa $25 \%(0,13 \mathrm{~g})$ der eingeführten Substanz in Form von Aetherschwefelsäuren ausgeschieden wurden.

Es sollen zum Schluss zwei Versuche erwähnt werden, in denen das Carbostyril subcutan verabreicht wurde (ebenfalls in Emulsion). Das eine Kaninchen erhielt $0,5 \mathrm{~g}$, das andere in drei Tagen im Ganzen $1,30 \mathrm{~g}$ Carbostyril. In beiden Fällen stellten sich die schon beschriebenen Lähmungserscheinungen ein; das erste Thier ging zu Grunde, das andere erholte sich bald. Die gesammelten Harne waren optisch inactiv, gaben nach dem üblichen Isolirverfahren keine Krystalle der gepaarten Glycuronsäure, auch konnte solche durch die Reductionsprobe nicht nachgewiesen werden. 


\section{Kynurin.}

$\mathrm{C}_{9} \mathrm{H}_{7} \mathrm{NO}\left(+3 \mathrm{H}_{2} \mathrm{O}\right)$. Das Versuchsmaterial wurde nach Schmiedeberg und Schultzen ${ }^{1}$ ) durch Schmelzen der reinen Kynurensäure dargestellt. Nach Monate lang fortgesetztem Sammeln von Kynurensäure gelang es uns, etwa $10 \mathrm{~g}$ reines Kynurin zu gewinnen. Die Base krystallisirt in glänzenden, harten Prismen, bei plötzlichem Ausfallen in feinen Nadeln. Die Substanz hat den verlangten Schmelzpunkt von $201^{\circ} \mathrm{C}$. Sie ist löslich in Wasser, leichter in Alkohol. Auf Zusatz von $\mathrm{Fe}_{2} \mathrm{Cl}_{6}$ entsteht eine carminrothe, von Millon's Reagens eine gelbgrüne Färbung. Reines Kynurin gibt die von Jaffée ${ }^{2}$ ) für Kynurensäure angegebene Reaction.

Nach A. Schmidt, der einem Kaninchen 0,5 g Kynurin subcutan injicirte, wurde die Substanz unverändert ausgeschieden.

Nach dem negativen Ausfall der subcutanen Carbostyrilversuche (in Bezug auf gepaarte Glycuronsäure) schien es uns vortheilhafter, das Kynurin per os zu verabreichen. So erhielten zwei Kaninchen $9 \mathrm{~g}$ Kynurin in wässeriger Lösung, zuerst in Dosen von $0,5 \mathrm{~g}$, dann aber $1 \mathrm{~g}$. Dabei zeigten die Thiere gar keine krankhafte Erscheinungen; sie nahmen sogar an Gewicht zu. Somit kann das Kynurin im Vergleich zu dem isomeren Carbostyril für das Kaninchen als vollkommen unschädlich bezeichnet werden. Nicht so aber für den Frosch. Ein Frosch, der 0,05 g Kynurin subcutan erhielt, zeigte genau dieselben Lähmungserscheinungen bei lange Zeit normaler Herzthätigkeit, wie solche nach Carbostyril beobachtet wurden.

Der Harn der mit Kynurin verfütterten Kaninchen dreht die Polarisationsebene nach links, und reducirt alkalisches Kupferoxyd nach verhältnissmässig kurzem Kochen mit $10 \% \mathrm{HCl}$.

Ein Theil des gesammelten Harnes wurde nach dem von Schmidt angegebenen Verfahren bearbeitet. Der Harn wurde eingedampft, mit Alkohol erschöpft, der Alkohol verdunstet, der Rückștand mit schwefelsaurem Aether geschüttelt und der

1) Schmiedeberg u. Schultzen, Ann. d. Chemie u. Pharm., 164, Seite 155.

2) Jaffé, Zeitschr. f. phys. Chemie, Bd. VII, S. 399. 
saure, wässerige Rückstand dem Krystallisiren überlassen. Es schieden sich dabei spärliche nadelförmige Krystalle $a b$, welche auf Zusatz von $\mathrm{Fe}_{2} \mathrm{Cl}_{6}$ zuerst die für Kynurin charakteristische rothe Färbung zeigten. Aber schon nach wenigen Secunden ging die rothe Farbe in grün, später in tiefblau über. Der Zusatz von $\mathrm{HCl}$ beschleunigt den Farbenumschlag, auf viel $\mathrm{HCl}$ verschwindet die blaue Farbe, kommt aber bei Verdünnung der Flüssigkeit mit Wasser wieder zum Vorschein. Die Rothfärbung des Kynurins auf Zusatz von $\mathrm{Fe}_{2} \mathrm{Cl}_{6}$ bleibt dagegen Tage lang unverändert, und auf Zusatz von $\mathrm{HCl}$ verblasst sie, ohne in blau überzugehen. Somit waren die Krystalle nicht Kynurin.

Die Hauptmenge des Harnes wurde nach dem oben beschriebenen Bleiverfahren bearbeitet. Der grösste Theil der links drehenden Substanz befand sich auch diesmal im basischen Bleiniederschlag, ein kleiner aber auch im Filtrat des Letzteren, aus dem er dann auf Zusatz von viel Ammoniak gefällt wurde. Die nach Entbleien des basischen Bleiniederschlages gewonnene Flüssigkeit färbte sich beim Einengen auf dem Wasserbade dunkel und wurde trübe. Beim Filtriren wurde das Filtrirpapier rosaroth gefärbt. Nachdem auch bei starkem Einengen keine Krystallisation eintrat und eine Zersetzung der Substanz zu befürchten war, schritten wir zur Darstellung des Kalisalzes der vermutheten gepaarten Glycuronsäure. Beim Einengen der wässerigen Lösung des Kalisalzes, das mit Hülfe des Barytsalzes gewonnen war, schieden sich nadelförmige Krystalle aus, die durch wiederholtes Umkrystallisiren und Behandeln mit Thierkohle gereinigt wurden. Das Kalisalz schmilzt unter Zersetzung bei $258-260^{\circ} \mathrm{C}$. Die wässerige Lösung reagirt amphoter und gibt auf Zusatz von Eisenchlorid dieselbe Farbenreaction, wie die erwähnten aus dem alkoholischen Harnextract gewonnenen Krystalle. Das Kalisalz reducirt alkalisches Kupferoxyd direkt nicht, wohl aber nach Kochen mit verdünnter $\mathrm{HCl}$. Aus der Spaltungsprobe schieden sich nach längerem Stehen nadelförmige Krystalle aus, welche die Kynurinreaction (beständige Rothfärbung auf Zusatz von $\mathrm{Fe}_{2} \mathrm{Cl}_{6}$ ) geben. Dieses Spaltungsprodukt scheint also Kynurin zu sein. Aus dem entbleiten ammoniakalisch-basischen Bleiacetat- 
niederschlage wurden nach starkem Einengen nadelförmige Krystalle gewonnen, die sich bezüglich der Eisenchlorid- und Reductionsprobe dem Kalisalz ganz analog verhielten. Ihre Lösung reagirte aber sauer. Wir hielten diese Substanz für die freie gepaarte Glycuronsäure; sie konnte wegen ihrer geringen Menge nur zu den erwähnten Reactionen benutzt werden. Zur Analyse etc. wurde das Kalisalz verwendet.

Auf Grund der beobachteten Eigenschaften (Linksdrehung, Reduction nach Kochen mit Salzsäure), sowie der Analogie der beiden anderen Oxychinoline (o-Oxychinolin, Carbostyril) schien es uns in hohem Grade wahrscheinlich, dass auch in diesem Falle eine Verbindung von Kynurin und Glycuronsäure vorlag. Da sonstige Bestandtheile ausser $\mathrm{C}, \mathrm{H}$ und $\mathrm{N}$ nicht vermuthet wurden, so verzichteten wir vorläufig - um an Substanz zu sparen (wir besassen im Ganzen etwa 0,6 g Kalisalz) - auf qualitative Proben und führten gleich die Elementaranalyse des Kalisalzes aus.

Die lufttrockene Substanz nahm beim Stehen über $\mathrm{H}_{2} \mathrm{SO}_{4}$ im Vacuum kaum etwas an Gewicht ab. Eine weitere Gewichtsabnahme wurde auch nach Trocknen bei $100^{\circ}$ nicht beobachtet.

Zum Zwecke der Analyse wurde die Substanz im Luftbade bei $100^{\circ}$ bis zur Gewichtsconstanz getrocknet.

I. $0,1862 \mathrm{~g}$ lieferten . . . 0 0,0459 $\mathrm{g} \mathrm{H}_{8} \mathrm{O}$

$$
0,2718>\mathrm{CO}_{2}
$$

II. 0,1615 * verbrauchten $\ldots 5,5 \mathrm{ccm} .1 / 10 \mathrm{~N}-\mathrm{HCl}$

III. 0,1067 , lieferten ..... 0,0314 $\mathrm{g} \mathrm{K}_{\mathbf{2}} \mathrm{SO}_{4}$.

$\begin{array}{cc}\text { Gefunden: } & \mathrm{C}_{15} \mathrm{H}_{16} \mathrm{NO}_{8} \mathrm{~K} \text { verlangt: } \\ \mathrm{C}=39,81 \% & \mathrm{C}=47,75 \% \\ \mathrm{H}=2,74 \% & \mathrm{H}=4,24 \% \\ \mathrm{~N}=4,77 \% & \mathrm{~N}=3,71 \% \\ \mathrm{~K}=13,19 \% & \mathrm{~K}=10,37 \% .\end{array}$

Die Analysenzahlen stimmen also in keiner Weise zu der angenommenen Formel. Besonders die niedrigen Werthe von $\mathrm{C}$ und $\mathrm{H}$ liessen die Anwesenheit eines weiteren Bestandtheiles in dem Molekül vermuthen.

Eine kleine Menge des Kalisalzes wurde mit Salpeter und Soda zerstört und auf Schwefel und Phosphor geprüft. Phosphor wurde nicht gefunden, dagegen eine ansehnliche 
Menge von Schwefel. Dieser war als gebundene Schwefelsäure im Molekül vorhanden, denn die mit Essigsäure und $\mathrm{BaCl}_{2}$ versetzte klare Lösung des Kalisalzes trübte sich beim Kochen mit HCl. Eine Verunreinigung mit gepaarten Schwefelsäuren konnte ausgeschlossen werden, da das Kalisalz wiederholt umkrystallisirt und mikroskopisch völlig einheitlich war.

Der Körper ist also complicirter zusammengesetzt, als wir vermutheten. Zur Aufklärung dieser Zusammensetzung ist weiteres Material nöthig, mit dessen Herstellung wir beschäftigt sind.

Zum Schluss sei es mir gestattet, Herrn Prof. Thierfelder für die Anregung $\mathrm{zu}$ dieser Arbeit und für die Unterstützung, die er mit Rath und That mir gütigst zu Theil werden liess, meinen verbindlichsten Dank auszusprechen. 